\title{
Medicina, higiene mental y saber $p s i$ en la construcción de la maternidad y la infancia en la Argentina: un análisis a través de la revista Madre y Niño (1934-1935)*
}

\author{
Medicine, Mental Health and Psy Knowledge in the \\ Construction of Maternity and Childhood in Argentina: an \\ Analisys through the Journal Madre y Niño (1934-1935)
}

Recibido: diciembre 12 de 2014 | Revisado: enero 27 de 2014 | Aceptado: julio 17 de 2014

\author{
ANA BRIOLOTTI ** \\ Sebastián Matías BeníteZ **** \\ Universidad Nacional de La Plata, Argentina
}

doi.org/10.11144/Javeriana.upsy13-5.mhms

Para citar este artículo: Briolotti, A., \& Benítez, S. M. (2014). Medicina, higiene mental y saber psi en la construcción de la maternidad y la infancia en la Argentina: un análisis a través de la revista Madre y Niño (1934-1935). Universitas Psychologica, 13(5), 1709-1719. http://dx.doi.org/10.11144/Javeriana.upsy13-5.mhms

- El presente trabajo se encuentra enmarcado en un Proyecto de Investigación más amplio que se propone indagar las relaciones entre el orden social y la producción de conocimiento y prácticas de la psicología en diversos campos profesionales: la educación, la criminología, la clínica médica y la interpretación ensayística histórico-social. El estudio se centra en la Argentina, desde fines del siglo XIX hasta mediados del siglo XX. En particular, nos interesa destacar las relaciones entre los actores que investigan en psicología desde un lugar dentro del orden social; los usos de la psicología en diversas prácticas profesionales; y la inserción de estos discursos y prácticas para legitimar un entramado social más amplio.

** Contacto principal para correspondencia editorial: correo electrónico abriolotti@psico.unlp.edu.ar

***** sbenitez.psi@gmail.com

\section{RESUMEN}

El presente trabajo se propone analizar el modo en que los saberes psi definieron ciertas concepciones en torno a la maternidad y la infancia, difundidas a través de la revista Madre y Niño, publicada en la Argentina a mediados de la década de 1930. La utilización de la psicología contribuyó a la legitimación del saber médico sobre la infancia a partir de definir su especificidad y combatir las nociones y prácticas populares, consideradas perjudiciales y anticientíficas. En la Argentina, la articulación de tales discursos y prácticas ha configurado el proceso de disciplinarización e institucionalización de la psicología y su utilización por parte de los saberes médicos como una estrategia de intervención social.

Palabras claves:

historia de la psicología; medicina infantil; higiene mental; divulgación científica

\section{A B S T R A C T}

The aim of this article is to analyze how the psychological knowledge defined conceptualizations about motherhood and childhood in several articles written by doctors for the magazine Madre y Niño, published in Argentina in the mid-thirties. The use of psychological knowledge about the bond between mothers and sons, would have provided scientific legitimation to Medicine in order to define its own field of expertise. Psychology in Argentina was able to develop a common ground with other fields of knowledge such as Medicine, taking into account its attempt to differ from other popular knowledges, considered harmful and non-scientific. This field of expertise can be framed in the process of disciplinarization of psychological knowledge and can be described as a tendency towards a social intervention. Keywords:

history of psychology; childhood medicine; mental hygiene; dissemination 
Se entiende por saberes psi todos aquellos discursos, disciplinas y prácticas en los que se enmarca la producción de trabajos científicos y de difusión masiva respecto de lo psíquico (Dagfal, 2009). De esta manera, puede establecerse un parámetro más inclusivo en el que se articulan tres áreas disciplinares distintas que se ocupan de lo psicológico: la psicología, la psiquiatría y el psicoanálisis. En la Argentina, dichas disciplinas se articularon estableciendo límites difusos y móviles respecto de la producción de conocimiento sobre los fenómenos psíquicos. Por otra parte, durante el período estudiado, la producción de saberes psicológicos se desarrolló en el ámbito de otras disciplinas y en superposición con ellas. En efecto -en el contexto previo a la creación de las carreras de Psicología y los comienzos de la Psicología como profesión ${ }^{1}$ - la criminología, la pedagogía y la medicina fueron áreas de conocimiento que recibieron significativos aportes por parte de los saberes psi, lo cual permitió legitimar numerosas prácticas de intervención. Mientras que la disciplina médica en la Argentina se consolidó a fines del siglo XIX, los saberes psi se desarrollaron a partir de la matriz del higienismo y la medicina, para dar respuesta a problemas de índole política social, cultural y epistemológica. Los saberes sobre lo psicológico, entonces, tomaron como una grilla interpretativa a los conocimientos médicos, en tanto que delimitaron los fenómenos de lo normal y lo patológico a partir de esa demarcación (Talak, 2014). De este modo, los saberes psi pudieron desplegarse a partir de su relación con los campos disciplinares de la medicina, la pedagogía y la criminología, al tiempo que se favoreció la utilización de un vocabulario psicológico en la interpretación de fenómenos sociales, culturales, políticos y de los criterios de normalidad-enfermedad.

El niño y la relación madre-hijo se constituyeron como objetos privilegiados de intervención de los

1 La creación de las primeras carreras de Psicología en la Argentina tuvo lugar entre 1956 y 1959. Las mismas se desarrollaron en la Universidad del Litoral, en Rosario (1956), en la Universidad de Buenos Aires (1957), en la Universidad Nacional de La Plata, en la Universidad Nacional de Córdoba y en la Universidad Nacional de San Luis (1958) y en la Universidad Nacional de Tucumán (1959) (Dagfal, 2012) profesionales médicos y, por lo tanto, se erigieron como nuevos objetos de estudio de estos saberes (Talak, 2005). Asimismo, la disciplina médica tuvo un lugar central en la recepción de discursos y prácticas psicológicas que resultaron útiles, entre otras cosas, para comprender y abordar la problemática de la anormalidad infantil (Borinsky \& Talak, 2005).

N. Rose (1996) ha señalado que desde fines del siglo XIX la medicina se nutrió de teorías y técnicas psicológicas para comprender e intervenir sobre ciertos fenómenos en su intento de dar solución a diversos problemas en la esfera individual y poblacional. En ese sentido, ha contribuido al proceso de disciplinarización de la psicología, por el cual el saber psicológico obtuvo el reconocimiento y la legitimación de la comunidad científica. Asimismo, el uso de la psicología por parte del saber médico de la época pondría de manifiesto su función reguladora respecto de intereses socioculturales diversos, múltiplemente determinados por proyectos políticos, sociales, económicos, epistemológicos, entre otros. De este modo, la institucionalización y disciplinarización de los saberes psi no pueden ser pensadas sin su articulación con políticas de intervención más amplias que utilizan los conceptos psicológicos para legitimarse y que ponen de manifiesto tanto la dimensión epistemológica como la dimensión tecnológica de los mismos.

Estos enfoques serían solidarios con los planteos que sostienen que la infancia no es una categoría descriptiva de una fase evolutiva natural, sino que se trata de una construcción singular, caracterizada por su contingencia, su historicidad y su relación con la política y la cultura (Cosse, Llobet, Villalta, $\&$ Zapiola, 2011). De igual modo, la maternidad puede ser pensada como un fenómeno cultural que cambia a lo largo del tiempo y en el que los aspectos materiales y simbólicos se entrecruzan y se influyen mutuamente (Nari, 2004). Retomando estos desarrollos, el trabajo se propone identificar una serie de ideas y valoraciones en torno a la infancia y la maternidad vehiculizadas, en este caso, a través del discurso médico, que recurría a ciertos saberes psicológicos cuando los temas abordados correspondían a la esfera moral, al carácter, a las relaciones humanas, entre otros. 
La utilización de los saberes psi por parte de la medicina implicaba necesariamente una dimensión política que contribuía a la legitimación del saber médico sobre la infancia, a partir de definir su especificidad y combatir las nociones y prácticas populares, consideradas perjudiciales y anticientíficas. Como podrá apreciarse, las revistas de divulgación del estilo de Madre y Niño cumplieron un importante papel en esta empresa de aculturación de los padres emprendida por los médicos. Tal como ha señalado M. Freire (2007) para el caso de Brasil, el ideal de la maternidad científica fue el blanco del discurso médico en las revistas femeninas y se constituyó como un símbolo de estatus que acercaba a las mujeres al mundo científico y racional de los hombres. Por otra parte, este tipo de intervenciones, así como también el foco puesto en la acción de las madres sobre el cuerpo infantil, puso de manifiesto la importancia que cobraba la acción temprana en la prevención de la futura degeneración. De este modo, la profilaxis y la acción médica temprana apuntaron a regular los comportamientos de los individuos de una sociedad, en función del sostenimiento del orden social (Silva Junior \& Monteiro García, 2010).

\section{Infancia, maternidad y familia en los años treinta: saber experto y orden social}

$\mathrm{Al}$ momento de pensar el rol del saber médico en la construcción de la infancia y la maternidad es pertinente considerar ciertas ideas y problemas propios de la época abordada, por cuanto ponen de manifiesto las valoraciones que influyeron en la constitución y difusión de un saber experto sobre estos temas.

Desde fines del siglo XIX, el problema de la cantidad y calidad de la población argentina fue una preocupación constante de las clases dirigentes. En este marco, durante las primeras décadas del siglo XX el Estado argentino profundizó su injerencia en acciones de asistencia y protección materno-infantil, que hasta ese momento eran emprendidas por el sector privado. Este hecho permitió la ampliación de la red de instituciones destinadas a tales fines y planteó la necesidad de organizar a nivel nacional la protección materno-infantil. Un primer paso en ese sentido se concretó en 1923 con la creación de la Sección de Asistencia y Protección a la Maternidad y la Infancia en el seno del Departamento Nacional de Higiene. Unos años más tarde, en diciembre de 1936, se sancionó la Ley 12341 que preveía la creación de la Dirección de Maternidad e Infancia. Una de las finalidades de dicho ente era promover el perfeccionamiento de las generaciones futuras, para lo cual se proponía, entre otros objetivos, la difusión de los postulados de la puericultura y la higiene infantil y la vigilancia del niño desde su nacimiento, a través de libretas o fichas sanitarias (Novick, 2004).

En el marco de estas preocupaciones -que remitían en última instancia al futuro de la nacióndurante la década de 1930 la infancia comenzó a ser pensada en relación con la familia, hecho que delineó el perfil de las políticas de protección materno-infantil. Así, se produjo un movimiento de pasaje de la penalización del abandono infantil al intento de mejorar las condiciones de vida de la madre y la familia, para mejorar de ese modo las condiciones de vida del niño. Asimismo, el contexto de crisis sociopolítica de esos años promovió la revalorización del modelo de familia nuclear y patriarcal como espacio de socialización de los niños y de contención de posibles conflictos sociales. Estas ideas decantaron en la importancia atribuida al rol materno en la crianza de los niños y al ámbito familiar como espacio propicio para forjar individuos aptos para respetar la autoridad y poder convivir en sociedad (Cosse, 2005). Y en este punto, el discurso médico ocupó un lugar nada desdeñable en el reforzamiento de la representación ideal de la familia y en la transmisión de un saber vinculado con la crianza de los niños cuyo énfasis recaía en la importancia del temperamento y la actitud de los padres para moldear el alma infantil. En ese sentido, y como se mencionó anteriormente, el dominio y la difusión de ciertos conocimientos psicológicos habrían contribuido al sostenimiento de un determinado orden social reivindicado por las elites políticas e intelectuales.

En el caso de la revista analizada en este trabajo, su publicación y dirección estuvo a cargo de 
Pedro Rueda (1880-1962), médico pediatra nacido en el pueblo de Simoca, en la provincia de Tucumán y cuyo trabajo se desplegó principalmente en la ciudad de Rosario, situada al sur de la provincia de Santa Fe. En 1919 fundó La Casa del Niño, institución encargada del cuidado de la infancia que cumplió un papel destacado en la atención en salud de Rosario, puesto que sus instalaciones sirvieron de base al Instituto de Lucha Antipoliomielítica y Rehabilitación del Lisiado. En 1929, creó en la Facultad de Medicina de la Universidad del Litoral la Cátedra de Puericultura que tuvo a su cargo, en un intento de llevar a cabo un proyecto de formación de los médicos respecto de la infancia temprana.

Tanto en su rol de médico puericultor como de formador de otros médicos, el papel de Pedro Rueda y de la revista Madre y Niño puede entenderse como un ejemplo de la serie de estrategias que intentaron consolidar un saber científico acerca de la infancia temprana y las prácticas de crianza, para lo cual la medicina recurrió a ciertos saberes provenientes del campo psi. A los fines del presente trabajo, se analizarán los números correspondientes a los primeros dos años de publicación, en tanto allí se explicitan sus objetivos, dando cuenta de la articulación entre el saber médico, la psicología y el movimiento del higienismo. Si bien la revista recibía colaboraciones de diversas figuras de la medicina local, la gran mayoría de los artículos eran escritos por el propio Rueda y respondían a sus ideas, lo cual se aclaraba en algunos de los editoriales de la revista.

Dirigida a la crianza y cuidado de los niños menores de dos años, Madre y Niño se planteaba como un deber "constituir, desde hoy en más, el verdadero y celoso guardián de la salud del niño y el consuelo de toda madre afligida." (Madre y Niño [MN], 06/1934, p. 3). Es preciso señalar que, si bien desde fines del siglo XIX las pautas de cuidado y crianza de los niños se transmitían a través de los manuales de puericultura (en su mayoría escritos por médicos varones), a partir de la década de 1930, comenzaron a editarse revistas del estilo de Madre y Niño, que procuraron agilizar la transmisión de dichos conocimientos recurriendo a un lenguaje más llano y a un estilo más fluido que el de los manuales de puericultura (Nari, 2004). En efecto, y con la fi- nalidad de adaptarse "a la mentalidad de cualquier lector medianamente preparado" (MN, 06/1934, p. 3), Madre y Niño se proponía divulgar una serie de conocimientos considerados imprescindibles para la crianza, utilizando un lenguaje sencillo y claro, despojado de toda clase de términos que pudiesen oscurecer la comprensión. Dichos consejos tomaban la forma de artículos breves intercalados con poemas, cuentos y secciones humorísticas, además de numerosas publicidades de medicamentos y productos para el bebé.

La revista se dirigía a las mujeres en general y a las madres en particular, y pretendía tratar los problemas e interrogantes que se planteaban cotidianamente en el hogar (MN, 06/1934). En este punto es interesante destacar las valoraciones que la propia publicación atribuía a sus lectores, ya que, según se afirmaba, las personas interesadas en la lectura de Madre y Niño "demuestran tener un alto grado de cultura, una clara visión del deber de colaborar al esfuerzo colectivo por aliviar y mejorar las condiciones de la humanidad" (MN, 06/1935, p. 36). Puede pensarse que estas virtudes atribuidas al lector habrían contribuido indirectamente al ennoblecimiento de la tarea del médico, al tiempo que se corresponderían con toda una serie de valoraciones en torno al discurso higiénico, que asimilaron el respeto por la higiene a una práctica civilizatoria y socializadora, teñida de preceptos morales (Armus, 2007). Precisamente, la revista adoptaba los postulados del higienismo en el marco de la importancia atribuida por dicho movimiento a la profilaxis y al diagnóstico temprano. A continuación se plantean algunas cuestiones fundamentales para pensar el papel desempeñado por la higiene en este tipo de intervenciones que tomaban por objeto al binomio madre-hijo.

\section{Higienismo e higiene mental: la importancia de la profilaxis y la intervención temprana}

El papel de Rueda en las políticas públicas respecto de la salud en Rosario no puede desligarse de una serie de proyectos que también tuvieron su eje en dicha ciudad. Por un lado, es de destacar la figura de 
Lanfranco Ciampi (1885-1968), médico psiquiatra que llegó a la Argentina desde Roma en 1922 para dirigir el Instituto Médico-Pedagógico en Rosario. Fue creador de la Cátedra de Psiquiatría Infantil de la Facultad de Ciencias Médicas y se convirtió en el director del Hospicio de Alienados de esa ciudad. Por otra parte, Gonzalo Bosch (1885-1962), médico psiquiatra, fue profesor titular de la cátedra de Psiquiatría de Rosario entre 1922 y 1930, hasta la creación de la Liga Nacional de Higiene Mental y su designación como director del Hospicio de las Mercedes en Buenos Aires (Gentile, 1998).

Dependiendo primeramente de la Sociedad de Neurología y Psiquiatría, la Liga Nacional de Higiene Mental intentó constituirse sin éxito en 1922, así como en 1924 a partir de las experiencias de Gonzalo Bosch en Estados Unidos. En un marco fuertemente impregnado por la neurología, la higiene mental se conformaría paulatinamente al interior del campo psiquiátrico, más precisamente dentro de los parámetros de la medicina social. La higiene mental fue, al igual que en Estados Unidos, un movimiento amplio que se inició en torno a las instituciones de internación psiquiátrica pero cuyo eje fue virando al campo de la profilaxis y la intervención social (Klappenbach, 1999). Esta matriz de intervenciones individuales, articulada con una perspectiva poblacional, permitió diferenciar una función privilegiada del movimiento de higiene mental. No se trataba simplemente de delimitar las condiciones ambientales que permitiesen minimizar la aparición de enfermedades, sino que se ponía el eje en "la noción de profilaxis [que] aludía a las intervenciones que buscaban a través de medios selectivos desterrar en el presente los elementos perniciosos para la sociedad futura" (Talak, 2005, p. 564).

La profilaxis mental insistía, en ese sentido, en el diagnóstico precoz. Publicaciones como Madre y Niño, ponían especial énfasis en la detallada observación de la relación materno-filial por parte de los médicos, así como un vínculo de alianza entre madre y médico que permitiera la intervención temprana. Este tipo de intervenciones procuraban retardar la aparición de la enfermedad mental, ya que no se dejaba de lado el papel determinante que podía tener la herencia. Desde una matriz evolucionista, que se desarrolló en virtud de las relaciones entre el saber médico y otras áreas de conocimiento como la biología y la filosofía, el higienismo formó parte de las estrategias epistemológico-intervencionistas en las que se articularon el problema de la enfermedad mental, del desarrollo infantil, del desarrollo de la nación y el de la evolución de la especie.

Junto con esta conceptualización, también se desarrolló la tesis del desencadenamiento "desadaptativo" de los trastornos psíquicos que, apuntaba a incluir las circunstancias familiares, laborales y aun políticas como factores destacados que, pensados como desencadenantes, delimitaban un espacio sobre el que el psiquiatra podía y debía intervenir (Talak, 2005, p. 596).

En función de estos objetivos, la revista Madre y Niño puede incluirse dentro de las estrategias de difusión de las ideas de la higiene a través de conferencias, carteles, folletos y multiplicidad de artículos de prensa dirigidos fundamentalmente a las madres, cuya función era propagar estas ideas en el seno familiar (Talak, 2005). Se destaca, en ese sentido, un artículo de Madre y Niño titulado "La higiene -ciencia hermana de la medicina- se puede dividir en dos partes" (MN, 05/1935, p. 20), en el que dicha doctrina se presentaba al público general de la revista. La higiene era definida como una disciplina que estudiaba las influencias del medio ambiental como "el suelo, el agua, la temperatura, la luz, la atmósfera, los microorganismos, la alimentación, el vestido y aún la higiene individual que es común a todos los individuos de la especie" (MN, 05/1935, p. 20). Asimismo, debía cumplir su función como una "Higiene Especial: ya sea de lo que surge de un estado o modalidad de su vida y que depende de la edad, oficio y condiciones [de vida de los hombres] o ya que ellas sean creadas por el hombre mismo" (MN, 05/1935, p. 20). En el marco de la higiene, el saber médico podía intervenir desde el amplio abanico de las condiciones ambientales como en la especificidad de las circunstancias etarias, sociales y económicas, tanto desde la intervención individual como la poblacional.

En lo que sigue, se plantearán algunas ideas respecto a las relaciones entre psicología y medicina 
infantil en el marco del proceso de profesionalización de esta última. A su vez, se analizará el modo como la revista concebía la psicología del niño y los aspectos en los que hacía hincapié, en función de ciertas ideas y valoraciones propias de la época.

\section{Medicina infantil y psicología: la importancia de conocer el alma del niño}

En la Argentina, al igual que en otros países de Latinoamérica, la institucionalización de los saberes y prácticas médicos se realizó en paralelo y de manera solidaria con la conformación del Estado nación a fines del siglo XIX. Dicho proceso dio lugar a la consolidación de una intelectualidad médica (González Leandri, 2006) que, en su pretensión de intervenir en el proceso de modernización propio de la época, tuvo un papel clave en la formulación y desarrollo de políticas sanitarias. Asimismo, se produjo un progresivo aumento de la injerencia del saber médico en la vida cotidiana y las costumbres de la población, a través de la prescripción de una serie de normas y pautas consideradas saludables. En el caso que nos ocupa, la puericultura se encargó de prescribir hábitos, prácticas y valores relativos al parto y la crianza con el fin de lograr la homogeneización de las prácticas y costumbres que circulaban entre las mujeres y que, ante la mirada médica, eran producto de la ignorancia y la negligencia (Nari, 2004)

Hacia los años treinta, si bien la medicina gozaba de un mayor prestigio y reconocimiento social, el campo profesional atravesó un período de crisis inescindible de la crisis política, económica y cívica que afectaba al país en esos años (Belmartino, 2005). En este marco, la autoridad médica se habría visto amenazada por el surgimiento de toda una serie de saberes parciales plasmados en técnicas diagnósticas (radiológicas, de laboratorio, entre otras), la especialización creciente y el fenómeno de la charlatanería y el curanderismo, cuyo auge en esos años venía a denunciar cierta "fisura" del saber médico (Klappenbach, 1995). En este marco, la psicología aparecía como un saber necesario para el médico, por su capacidad de responder a un doble propósito: "el de obtener estructuras conceptuales que iluminaran la compleja trama del enfermar, y el de disponer de herramientas aptas para enfrentar exitosamente los requerimientos de la intervención médica" (p. 193).

Cabe preguntarse qué particularidades asumió dentro de este proceso aquella rama de la medicina que se ocupaba del niño, y en la cual confluían la pediatría y la puericultura ${ }^{2}$. Al respecto, puede señalarse la relación de inherencia existente entre la delimitación de la medicina infantil como especialidad y la construcción de la categoría de infancia (Colángelo, 2011). En efecto, el reconocimiento de la pediatría y la puericultura como especialidades médicas supuso el recorte de un objeto de estudio que, por sus características singulares, volvía necesaria la intervención de los expertos. Así, la niñez era considerada una etapa transitoria y el niño un ser incompleto y maleable, con características físicas, fisiológicas y anímicas propias y diferentes de las del adulto.

Así delimitada, la medicina infantil buscó su legitimación hacia el interior del campo médico mostrando la especificidad de la niñez (desde el punto de vista de la salud y la enfermedad) y creando espacios académicos, asociaciones profesionales, eventos científicos y revistas especializadas. Hacia el exterior de ese campo, la pediatría y la puericultura obtuvieron reconocimiento a partir de su intervención en políticas públicas de atención de la salud y vigilancia higiénica de la población, de su lucha contra los saberes legos y de la creencia de la población en la eficacia del saber médico (Colángelo, 2011). En línea con lo planteado anteriormente, podría incluirse en este punto el recurso de la medicina infantil al saber psicológico con el fin de ampliar y profundizar la mirada sobre el niño, abarcando todos los aspectos del desarrollo y, en consecuencia, aumentando la eficacia de la intervención médica.

2 La pediatría, disciplina médica que se ocupa de las enfermedades del ser humano entre el nacimiento y hasta los 18 años de edad se diferenciaba de la puericultura, que había surgido en el marco de una preocupación por la salud de la mujer y el niño en la segunda mitad del siglo XIX, y que se ocupaba tanto del cuidado y la crianza de los niños sanos como de los niños considerados enfermos (Armus, 2007; Genta Mesa, 2006) 
La constitución de la puericultura como un campo dependiente de la medicina pero con cierta especificidad no solo se dio en la Argentina, sino también en otros países de Latinoamérica. Siguiendo el modelo francés, en México, los médicos eugenistas desarrollaron la puericultura como la cultivación científica del niño con una matriz evolucionista para dar cuenta de la importancia del vigoroso desarrollo de los niños. Siguiendo las ideas de A. Pinard, la preocupación por la infancia no se restringía a la ontogénesis del niño, sino también a los cuidados maternales en tanto se delimitaba el futuro de la nación (Stern, 1999). De hecho, la preocupación por el desarrollo de la nación funcionará como una matriz general de intervenciones por parte de las elites dirigentes latinoamericanas.

Ahora bien, iqué aspectos del psiquismo infantil se subrayaban en las páginas de Madre y Niño? Una de las cuestiones que se destaca es la presencia de una concepción de un alma infantil en la que priman dos rasgos: pureza y fragilidad. El espíritu del niño se caracterizaba por su gran susceptibilidad frente a los estímulos del medio exterior, los cuales podían llegar a alterar las funciones fisiológicas del organismo. En un artículo sobre el sueño del niño, Rueda (MN, 06/1934) afirmaba que durante los primeros tiempos de vida toda forma de excitación prolongada, como las visitas reiteradas y la concurrencia a teatros o cines, podían perturbar el reposo del niño. En un contexto de creciente modernización de las grandes ciudades y ampliación del acceso al consumo de bienes culturales, no es extraño que los médicos se preocupasen por advertir acerca de la nocividad de ciertos estímulos, especialmente cuando podían constituir la fuente de potenciales desórdenes morales y sociales. En efecto, el segundo número de la revista incluía un artículo dedicado a detallar las repercusiones del cinematógrafo en el alma infantil. Reaparecían allí las ideas de inocencia y pureza, a las cuales se añadía una predisposición natural a la curiosidad. Todo esto hacía del niño un ser fuertemente influenciable, en cuya mente podían imprimirse ideas de diversa índole, incluso contradictorias. Esto explicaba que el niño pudiese adquirir con igual facilidad hábitos buenos y costumbres reprobables. A su vez, se destacaba otro rasgo característico del psiquismo infantil: su poca capacidad de control, lo cual daba lugar a una aceptación pasiva de los estímulos:

Diríase que el alma del niño es un vehículo en marcha que perdiendo la dirección, puede chocar estrepitosamente con los innumerables obstáculos que se presentan a su paso, máxime si las fallas del poder frenador que le impone la deficiente constitución de su sistema nervioso, no permite detener la caída inevitable ante cualquier desperfecto del accidentado camino. (MN, 07/1934. p. 39)

Como puede verse, esta poca capacidad de control era atribuida a la inmadurez del sistema nervioso infantil. Este argumento se conjugaba con aquel que subrayaba el fuerte influjo ejercido por las tendencias instintivas. La presencia en el niño (sobre todo durante la primera infancia) de estos componentes heredados de las generaciones anteriores, permitía explicar desde la preferencia por juguetes que representaran seres vivos, hasta fenómenos como la irrupción del miedo inmotivado o la ira. Respecto del miedo inmotivado, uno de los artículos (MN, 07/1935: pp. 21, 26, 31, 40) lo conceptualizaba desde una óptica naturalista-evolucionista que, autorizándose en Darwin, Spencer y Preyer, obligaba a pensar en un influjo hereditario proveniente de los antepasados. El bagaje instintivo con el cual el niño llegaba al mundo estaba compuesto por emociones "simples y primitivas" que, en tanto tales, delineaban un perfil de la infancia en tensión con aquella imagen del niño inocente y puro. Aparecía aquí el niño como un déspota que, valiéndose del llanto y los caprichos, lograba sojuzgar a cualquier madre que fuese demasiado solícita.

La imagen del niño inocente y la del niño tirano coexistían en el discurso médico, lo cual se pone de manifiesto en los consejos de crianza. Si bien se aconsejaba respetar al niño en su singularidad, no castigarlo ni criarlo con extremo autoritarismo, no dejaba de señalarse hasta qué punto era fundamental mantener una actitud serena pero firme y educar al niño de modo tal de poner freno a las tendencias instintivas y transformarlo en un hombre capaz de vivir en sociedad. Este énfasis en la idea de niñez 
como etapa de socialización era parte importante de los discursos nacionalistas sobre la infancia que caracterizaron la década de 1930. En dichos discursos, el niño era concebido como un actor clave en el destino de la nación y, en tal sentido, se destacaba la importancia de la sujeción a la autoridad estatal además de la familiar y escolar (Carli, 2012).

El análisis de la difusión de estas estrategias y preceptos de crianza a través de una publicación como Madre y Niño permite reflexionar sobre el modo como el saber médico intentó legitimar su práctica basándose en los postulados del higienismo y cumpliendo un rol determinante en el sostén del orden social establecido. En este sentido, podría pensarse que el énfasis en ciertos aspectos de la psicología del niño y las consecuentes prescripciones para su crianza, reflejan las ideas y valoraciones de un sector de la intelectualidad que reivindicaba las nociones de productividad, armonía social y virtud moral.

\section{Medicina social y familia: las madres como aliadas}

En Madre y Niño, la figura del médico se planteaba como un aliado de la madre que, en virtud de su saber, debía participar en prácticas de crianza tendientes a asegurar un normal desarrollo del niño. De este modo, las madres eran pensadas no solo como aquellas que se ocupaban de los niños, sino como un instrumento de la intervención médica sobre la infancia en pos del futuro de la nación. Bajo esta mirada, el médico y la madre cumplían un rol fundamental en el progreso de la patria (Freire, 2007). Por otra parte, la figura del padre no tenía un lugar preponderante en la publicación analizada pero eran los médicos, en general varones, quienes guiaban el modo en que debía constituirse el desarrollo infantil.

En uno de los artículos, titulado "Psicología de las Madres", Rueda sostenía que el médico y la madre, los dos agentes encargados del cuidado del niño, debían "marchar en la más íntima compenetración". De este modo, se planteaba que "una madre inteligente, solícita y disciplinada, es el mejor auxiliar del médico en la atención del niño sano o enfermo" (MN, 10/1934, p. 21) [destacado en el original].
El médico contaba con una amplia formación para interpretar las alteraciones del cuerpo y del espíritu. Con respecto a la madre, era fundamental que su mentalidad acompañara la tarea del galeno y armonizara con ella:

(...) si uno [el médico] debe poseer preparación clínica y psicológica; la otra debe tener una mentalidad suficiente para desterrar de su espíritu prejuicios que estorban seriamente a su acción y estar capacitada en forma, para observar con fidelidad los consejos que se le dicten. (MN, 10/1934, p. 21)

Así, la eficacia de la intervención médica parecía ser tributaria del dominio de esa "psicología de la madre", en la medida en que solo de ese modo era posible sortear las dificultades que podían presentarse en la atención del niño (MN, 10/1934).

Si la mujer-madre constituía el aliado fundamental del médico en el cuidado del niño, no era sin cierta reticencia. M. Nari (2004) ha señalado que las mujeres obreras sostenían una relación distante con los médicos, pero encontraban mayor cercanía con las parteras y auxiliares, mientras que las mujeres de estratos sociales medio-altos -a quienes iba dirigida la revista- podían sostener una mejor predisposición a la palabra del médico. Sin embargo, el problema respecto de la relación entre ciencia y saber popular no estaba exento de ambigüedades, frente a las cuales la revista claramente tomaba partido. En palabras del propio Rueda: “(...) cuantas (sic) veces a la salida del médico, sigue de inmediato la entrada del curandero, a quien suele brindársele mejor acogida. Es que una fuerte cohesión, asocia la rudimentaria psicología de las madres a la refinada simulación del taumaturgo" (MN, 10/1934, p. 21).

A partir de los consejos de los médicos en la publicación analizada, pueden verse las tensiones entre este ideal y las prácticas concretas que eran claramente impugnadas por parte de los médicos. El saber experto legitimaba prácticas consideradas salubrígenas, que permitían el desarrollo normal de la relación madre-hijo, por fuera de la transmisión intergeneracional de ciertos saberes populares. La presencia de dichos saberes, que a los ojos de la revista no eran sino prejuicios erróneos, reclamaba 
la lucha tenaz del médico en su trato cotidiano con "la polícroma sociedad de un pueblo múltiple; receptáculo de las más bizarras creencias y donde poca o ninguna acción, intenta destruir esos falsos conceptos" (MN, 10/1934, p. 21).

Si, por un lado, ser madre formaba parte de la naturaleza femenina ${ }^{3}$ también debía tenerse en cuenta el rol de la educación de esta misma naturaleza maternal. La tensión entre la idea de maternidad como instinto y como algo que debe aprenderse puede apreciarse en diversos artículos de la revista. Dicha ambigüedad se planteaba, por ejemplo, en lo referido por los médicos como una sensación de falta de preparación que se apoderaba de la mujer ante la inminencia de la maternidad. Sin embargo, esta era la función para la cual había nacido (MN, 06/1934). Y era precisamente esta falta de preparación de las madres la que impulsaba la necesidad de

(...) penetrar continua y pacíficamente en el hogar, dando en dosis metódica y proporcionada, todas aquellas nociones que la diaria y muchas veces imprevista contingencia hace necesario para evitar un mal y otras para aminorar un desarreglo que la despreocupación, puede transformar en irreparable pérdida. (MN, 06/1934, p. 3)

El médico, cuyo conocimiento respecto de la psicología de la madre debía ser indispensable, también ocupaba el rol de educador de las prácticas maternales. Si la revista estaba dirigida a un público culto y educado, puede entenderse la importancia de la escuela para preparar el espíritu de las madres. Sin embargo, el impacto de sus lecciones de puericultura daba lugar a "verdaderas batallas" entre hijas formadas en puericultura y madres que

3 Tal como lo ha analizado M. Nari (2004), entre 1920 y 1940 puede pensarse un proceso de biologización de la maternidad en la Argentina, en tanto se erige como el objetivo último de la realización femenina. El resultado más claro habría sido la conformación del binomio madre-hijo como una unidad funcional indisoluble al consolidarse una serie de saberes, políticas y prácticas respecto del vínculo entre la madre y el niño. Esta consolidación, permitió el desarrollo de la puericultura intrauterina, más ligada a un programa eugenésico, y de la puericultura extrauterina, orientada a consolidar el binomio madre-hijo. porfiaban en sus "atávicas, incorregibles y dañosas prácticas" (MN, 10/1934, p. 22).

El recorrido realizado hasta aquí ha permitido ilustrar el modo como los saberes psi fueron utilizados en el campo de la medicina a través de intervenciones tempranas sobre la primera infancia y las prácticas de crianza. En ese sentido, se conformó un entramado social específico a partir de discursos y prácticas del saber experto que legitimaba ciertas pautas de relación entre madre e hijo, en función de su potencial salubridad a nivel individual y poblacional.

\section{Comentarios finales}

A lo largo de los primeros números de la revista Madre y Niño, publicados y editados por el médico Pedro Rueda, es posible apreciar el modo como se articularon el discurso del higienismo y de la higiene mental para legitimar la intervención temprana del saber médico en la relación materno-filial. En este contexto, ciertas prácticas se habrían reconocido como salubrígenas a partir del recurso de los médicos al saber psicológico y marcando una diferencia con los saberes y prácticas populares, considerados no científicos. A su vez, el análisis de esta publicación permitiría dar cuenta del proceso de construcción de la primera infancia como objeto de intervención de esos saberes, así como también de la maternalización de las mujeres (Nari, 2004).

$\mathrm{Si}$ se consideran las ambigüedades observadas con respecto a la figura del niño (un sujeto frágil y necesitado de cuidados pero con la capacidad de manipular a su madre) y las consecuentes dificultades para su crianza, es posible pensar que dicha caracterización hacía necesaria la intervención del saber médico para una detección precoz que resguardase al niño de la enfermedad mental y de las anomalías del carácter. Este afán intervencionista del discurso médico, a partir de la utilización de los saberes psi, solo era posible a partir de la alianza con las mujeres-madres quienes debían confiar en la experticia del galeno. La relación entre el ejercicio de la maternidad y el lugar otorgado al saber médico, puede ser relevante para analizar el modo en que se plantean las intervenciones sobre la infancia en 
la actualidad. Asimismo, nos permite ver cómo funcionan las relaciones entre hombres y mujeres a partir del análisis del ejercicio de los roles parentales así como el lugar que se le da a los expertos en la intervención sobre la infancia temprana.

La revista Madre y Niño, entre otras publicaciones de la época, cumpliría una función estratégica como medio de difusión de los saberes psi. En el marco de las ideas del higienismo en la Argentina, formaría parte del proceso de legitimación de las intervenciones médicas al interior de la familia, en una época en la cual la incorporación de un saber "científico" sobre la crianza parecía ser fundamental para garantizar el futuro de las nuevas generaciones.

\section{Referencias}

Armus, D. (2007). La ciudad impura. Salud, tuberculosis y cultura en Buenos Aires, 1870-1950. Buenos Aires: Edhasa.

Belmartino, S. (2005). La atención médica argentina en el siglo XX: instituciones y procesos. Buenos Aires: Siglo XXI.

Borinsky, M., \& Talak, A. M. (2005). Problemas de la anormalidad infantil en la psicología y la psicoterapia. Buenos Aires: Facultad de Psicología, UBA.

Carli, S. (2012). Niñez, pedagogía y política: transformaciones de los discursos acerca de la infancia en la historia de la educación argentina (1880-1955). Buenos Aires: Miño y Dávila.

El cinematógrafo en el alma del niño. (1934, julio). Madre y Niño, 1(2), 30-32.

Colángelo, M. A. (2011). El saber médico y la definición de una "naturaleza infantil" entre fines del siglo XIX y comienzos del siglo XX en la Argentina. En I. Cosse, V. Llobet, C. Villalta \& M. Zapiola (Eds.), Infancias: políticas y saberes en Argentina y Brasil: siglos XIX y XX (pp. 101-121). Buenos Aires: Teseo.

Un concepto sobre las personas que leen "Madre y Niño". (junio, 1935). Madre y Niño, 2(1), 36.

Cosse, I. (2005, agosto). La infancia en los años treinta. Todo es Historia, 38(457), 48-54.

Cosse, I., Llobet, V., Villalta, C., \& Zapiola, M. C. (2011). Introducción. En I. Cosse, V. Llobet, C. Villalta \& M. Zapiola (Eds.), Infancias: políticas y saberes en Argentina y Brasil: siglos XIX y XX (pp. 11-28). Buenos Aires: Teseo.

Dagfal, A. (2009). Entre París y Buenos Aires. La invención del psicólogo (1942-1966). Buenos Aires: Paidós.

Dagfal, A. (2012) Historias de la psicología en la Argentina (1890-1966). Entre ciencia natural y disciplina del sentido. Ciencia Hoy, 126(21), 25-29. Recuperado de http://goo.gl/jcPRPV

Efemérides Rosarinas. (2002, 6 de agosto). La Capital, 138, 49.562. Recuperado de http://goo.gl/JpN22h

Freire, M. M. (junio, 2008). Ser mãe é uma ciência: mulheres, médicos e a construção da maternidade científica na década de 1920. História, Ciências, Saúde-Manguinhos, 15(Supl.), 153-171.

Genta Mesa, G. (2006) Anotaciones para una historia de la pediatría y la puericultura, Iatreia, 1 (3), 296 304. Recuperado de http://goo.gl/ReA5hKhttp://h

Gentile, A. (1998). La psiquiatría en Rosario. Temas de la Historia de la Psiquiatría Argentina, 5, 3-11. Recuperado de http://goo.gl/vvIIlEhttp:///h

González Leandri, R. (2006). La consolidación de una inteligentzia médico profesional en Argentina: 1880-1900. Diálogos. Revista Electrónica de Historia, 7(1), 36-78.

La higiene - ciencia hermana de la medicina- se puede dividir en dos partes. (1935). Madre y Niño, 1(12), 20.

Klappenbach, H. (1995). Psicología y campo médico. Argentina: años' 30. Cuadernos Argentinos de Historia de la Psicología, 1(1-2), 159-226.

Klappenbach, H. (1999). El movimiento de la higiene mental y los orígenes de la Liga Argentina de Higiene Mental. Temas de la Historia de la Psiquiatría Argentina, 10, 3-17. Recuperado de http://goo.gl/ FlFd5ohttp:///h

El miedo. (1935, julio). Madre y Niño, 2(2), 21, 26, 31, 40.

Nari, M. (2004). Políticas de maternidad y maternalismo político. Buenos Aires: Biblos.

Novick, S. (2004). Políticas del Estado argentino y rol de las mujeres: legislación referida a maternidad y enfermedades venéreas (1930-1943). Ponencia presentada en el Meeting of the Latin American Studies Association, Las Vegas, Nevada. Recuperada de http://goo.gl/R02Xor

Psicología de las madres. (1934, octubre). Madre y Niño, 1(5), 21-22. 


\section{MEDICINA, HIGIENE MENTAL Y SABER PSI EN LA CONSTRUCCIÓN DE LA MATERNIDAD Y LA INFANCIA EN LA ARGENTINA}

Rose, N. (1996). Inventing our selves: Psychology, power, and personhood. Cambridge: Cambridge University Press.

Rueda, P. (1934). Algunos temas que tratará "Madre y Niño". Madre y Niño, 1(1), 2.

Rueda, P. (1934). Nuestra presentación. Madre y Niño, 1(1), 3 .

Silva Junior, N. G. S., \& Monteiro García, R. (2010). Moncorvo Filho e algumas histórias do Instituto de Proteção e Assistência à Infância. Estudos e Pesquisas em Psicologia, 10(2), 613-632.

Stern, A. M. (diciembre, 1999) Responsible mothers and normal children: Eugenics, nationalism, and welfare in post-revolutionary Mexico, 1920-1940. Journal of Historical Sociology, 12(4), 369-397.

El sueño del niño. (1934, junio). Madre y Niño, 1(1), 15-16.

Talak, A. M. (2005). Eugenesia e higiene mental: usos de la psicología en la Argentina, 1900-1940. En M. Miranda \& Vallejo, G. (Comp.), Darwinismo social y eugenesia en el mundo latino (pp. 563-599). Buenos Aires: Siglo XXI de Argentina Editores.

Talak, A. M. (2014). La institucionalización de la psicología en la Argentina: saber universitario y usos sociales (1890-1920). Manuscrito Inédito. 
